\title{
RELAÇÕES ENTRE OS CONCEITOS “CULTURA, IDENTIDADE E DIFERENÇA” E O EXAME DE PROFICIÊNCIA EPPLE
}

\author{
RELACIONES ENTRE LOS CONCEPTOS "CULTURA, IDENTIDAD Y \\ DIFERENCIA” Y EL EXAMEN DE PROFICIENCIA EPPLE
}

\author{
CULTURE, IDENTITY AND DIFFERENCE RELATIONSHIP AND THE \\ PROFICIENCY EXAM EPPLE
}

Priscila Petian ANCHIETA ${ }^{1}$

\begin{abstract}
RESUMO: O presente trabalho discorre sobre o quanto aspectos como identidade, cultura e diferença são importantes para os ambientes de ensino e aprendizagem de línguas. Utilizando a definição de Woodward (2011) de que a identidade é marcada pela diferença, lançamos mão de aspectos que se fazem presentes em contextos de ensino e aprendizagem de língua estrangeira, quando aprendemos e utilizamos a língua do outro. Além disso, apresentamos um exame de proficiência denominado EPPLE, voltado para professores de línguas, e sugerimos a implementação de uma tarefa que aborde questões culturais, pois no mundo de pluralidade em que vivemos, precisamos formar professores de línguas que busquem aprimorar não apenas conceitos linguísticos e pedagógicos, mas também conceitos sobre a cultura, a identidade e a diferença.
\end{abstract}

PALAVRAS-CHAVE: Ensino e aprendizagem de línguas. Cultura. Identidade. Diferença. Tarefas.

RESUMEN: Se discurre, en el presente texto, en qué sentido los aspectos los aspectos como identidad, cultura y diferencia son importantes para los ambientes de enseñanza y aprendizaje de lenguas. A partir de la definición de Woodward (2011) de que la identidad está marcada por la diferencia, recurrimos a aspectos que se hacen presentes en contextos de enseñanza y aprendizaje de lengua extranjera, cuando aprendemos y utilizamos la lengua del otro. Además de eso, presentamos un examen de proficiencia denominado EPPLE, direccionado para profesores de lenguas, y sugerimos la implementación de una tarea que aborde cuestiones culturales, pues en el mundo de la pluralidad en el que vivimos, hay que formar profesores de lenguas que busquen perfeccionar no solo conceptos lingüísticos y pedagógicos, sino también conceptos sobre la cultura, la identidad y la diferencia.

PALABRAS CLAVE: Enseñanza. Aprendizaje. Cultura. Identidad. Diferencia. Tareas.

ABSTRACT: The present work discusses how aspects such as identity, culture and difference are important aspects in for language teaching and learning environments. Using Woodward's (2011) definition that identity is marked by difference, we

${ }^{1}$ Universidade Tecnológica Federal do Paraná (UTFPR), PR - Brasil. Professora do Magistério Superior na UTFPR. E-mail: pri_anchieta@yahoo.com.br. 
considered these aspects in foreign language teaching and learning contexts when we learn the laguage of others. In addition, we present a proficiency exam called EPPLE, aimed at language teachers, and we suggest the implementation of a task that addresses cultural issues, because we need to prepare language teachers that search not only for their linguistic and pedagogical knowledge construction, but also for their understanding about culture, identity and difference.

KEYWORDS: Language teaching and learning. Culture. Identity. Difference. Tasks.

\section{Introdução}

Apresentamos, neste artigo, uma discussão cujo objetivo principal é estabelecer sugestões e possibilidades de como podemos abordar o tema 'identidade e diferença' em um exame de proficiência para professores de Língua Estrangeira (LE) denominado EPPLE (Exame de Proficiência para Professores de Língua Estrangeira).

Iniciamos nosso trabalho embasando teoricamente o termo 'identidade', citando Woodward (2011) que afirma que a identidade, para existir, necessita da existência de uma segunda identidade. Como é o caso da identidade nacional brasileira que apenas existe devido à existência de outras identidades nacionais, como é o caso da identidade americana, japonesa, chinesa.

Em outras palavras, uma identidade depende de algo que existe fora dela, visto que se distingue por meio daquilo que ela não é. Sendo assim, Woodward (2011) chega à conclusão de que a identidade é marcada pela diferença. Com efeito, assumimos várias identidades, como a de sermos brasileiros, filhos, pais, mães, profissionais, que são estabelecidas baseadas em diferenças.

\section{A identidade e o professor de língua estrangeira}

Ao levarmos em consideração o professor de LE, é importante lembrar que ele também apresenta diversas identidades, inclusive a identidade nacional, ao ensinar a língua de outro país. Citamos aqui a identidade nacional, embora reconheçamos a existência das inúmeras identidades como, por exemplo, a identidade profissional, a identidade paterna, a identidade materna, conforme mencionamos anteriormente.

Tendo em vista que um professor de LE ensina uma dada língua que não é a sua língua materna, consideramos que, por meio da utilização da língua-alvo, ele não só abordará, mas também vivenciará a cultura do outro que se estabelece em oposição a sua cultura. 
Acreditamos que, para facilitar o processo de ensino e aprendizagem de línguas, é preciso que o professor seja proficiente na língua que ensina para que, por meio dela, possa trabalhar tanto aspectos linguísticos quanto aspectos culturais.

De acordo com Scaramucci (2000), a proficiência apenas deve ser definida a partir do contexto específico de ensino-aprendizagem vivenciado pelos alunos e dentro do qual ocorrem as possíveis interações. Assim, devem-se avaliar os aspectos da língua não de forma isolada e indivisível, mas de forma a abranger toda a sua complexidade, que é refletida na comunicação.

O conceito de proficiência representa, de modo geral, uma fonte de classificações para aprendizes proficientes e não proficientes. Porém, a autora afirma que a proficiência deve ser entendida como um termo dependente de outras variáveis, como o contexto de ensino e aprendizagem, suas características e suas metas, que podem também influenciar diretamente no desenvolvimento dos alunos.

Buscamos abordar neste artigo a proficiência linguístico-comunicativa do professor de língua estrangeira utilizada em ambientes de ensino e aprendizagem de línguas, como é o caso da sala de aula que abrange vários ambientes de interação, tanto presenciais, como as aulas presenciais, quanto ambientes online utilizados para fins de aprendizagem, como é o caso dos cursos online, dos e-mails, dos chats.

Nosso interesse de investigação gira em torno de dois pontos primordiais: a proficiência linguístico-comunicativa do professor e a forma com que ele utiliza sua proficiência para abordar questões de cultura, identidade e diferença.

\section{Cultura, identidade e diferença}

Considerando o fato de que vivemos em um mundo repleto de diferentes culturas, que se estabelecem por meio de suas diferenças, acreditamos que tais diferenças devem ser trabalhadas em ambientes de ensino e aprendizagem de línguas de forma a não excluir aquilo que é diferente, mas, sim, de vivenciar essas diferenças respeitando-as e conhecendo-as cada vez mais.

Hall (1990) afirma existirem dois tipos de identidades culturais: a primeira se constitui pela busca de uma comunidade por recuperar a verdade sobre seu passado, por meio da unicidade da história e de uma cultura partilhada, que afirma e reafirma a identidade. A segunda, na qual também acreditamos, aborda uma questão tanto de tornar-se quanto de ser. Esta abordagem não nega a identidade do passado, mas 
reconhece que reconstruímos nossa identidade no decorrer do tempo, visto que ela passa por constante transformação.

Sendo assim, ressaltamos a importância de professores de LE abordarem, por meio do ensino, a importância de não apenas apresentar estereótipos dos falantes de outras línguas, mas também de, por meio da língua, levar o aluno a refletir sobre a identidade do outro, levando em consideração os aspectos como transformação, diferença e pluralidade.

Desse modo, contribuiremos para que nossos alunos não apenas utilizem a língua estrangeira que estão estudando, mas também vivenciem essa língua de forma a compreender as diferenças que nela existem, não como uma forma de exclusão, mas como uma forma de vivenciar e compreender aquilo que vem de fora.

Assim, voltamos ao que Woodward (2011) diz: nossa identidade depende de algo que existe fora dela para existir, sendo assim, ao compreendermos a identidade nacional de um americano, por exemplo, poderemos compreender e estabelecer de forma mais clara a nossa própria identidade nacional.

Woodward (2011) afirma existirem duas abordagens da identidade. A visão essencialista, que considera a existência de um conjunto de características que todos sem exceção partilham e que não se alteram ao longo do tempo. Como se vivêssemos em um mundo que não se transforma. Já a segunda visão seria a não essencialista, que define a identidade focalizando as diferenças, assim como as características comuns e partilhadas entre povos de etnias tanto semelhantes como diferentes.

Visto que abordar questões sobre a identidade cultural em ambientes de ensino e aprendizagem de línguas é algo primordial no mundo de pluralidade em que vivemos, consideramos que a utilização de um exame de proficiência para professores de língua estrangeira que aborde tais questões pode contribuir para que nossos professores de línguas busquem aprimorar não apenas conceitos linguísticos e pedagógicos, mas também conceitos sobre cultura, identidade e diferença.

Concordamos com Silva (2011) quando ele discute que, pedagogicamente, as crianças e os jovens, nas escolas, seriam estimulados a entrar em contato, sob as mais variadas formas, com as mais diversas expressões culturais dos diferentes grupos culturais. Para essa perspectiva, a diversidade cultural é positiva e expressa, sob a superfície, nossa natureza humana comum. 


\section{O conceito de identidade e diferença no exame de proficiência para professores: EPPLE}

A proposta do grupo de pesquisa do EPPLE é a elaboração de um exame de proficiência em LE voltado para professores de línguas, considerando-se principalmente os perfis desses profissionais enquanto inseridos em cenários de ensino e aprendizagem de línguas. A partir de subsídios teóricos e experimentais, de dados da literatura e de testes de proficiência existentes tanto no âmbito do Brasil como do cenário internacional, buscamos subsídios para o aprimoramento e a implementação do exame.

De acordo com o site de divulgação (http://www.epplebrasil.org/), no decorrer do trabalho do grupo de pesquisa, tem-se buscado delimitar o construto do exame, os tipos de tarefas pertinentes à avaliação das habilidades linguísticas dos professores e os parâmetros a serem considerados na avaliação da proficiência linguística (PL) desejada para esses profissionais, para que atuem, por exemplo, na escola regular de Ensino Fundamental e Médio, no ensino superior e nos cursos particulares de idiomas.

O EPPLE avalia as habilidades de compreensão e produção linguística em LE, nas modalidades oral e escrita. Ele é composto por dois testes distintos: um de leitura e produção escrita e outro de compreensão auditiva e produção oral. Para melhor delimitar este trabalho, restringimo-nos em analisar o teste de leitura e produção escrita em língua inglesa.

No site de divulgação do exame existe um exemplar do teste de leitura e produção escrita de língua inglesa, no qual baseamos nossas reflexões. De acordo com o exemplar retirado do site, o teste de produção escrita é composto por textos baseados em temas que permeiam a linguagem utilizada por professores, além de questões em que o candidato deverá demonstrar domínio pedagógico, como é o caso da tarefa em que o avaliado deve corrigir erros gramaticais de um texto supostamente escrito por um aluno.

Toda a linguagem abordada no EPPLE volta-se para o ambiente de atuação do professor de língua estrangeira, buscando incorporar situações reais de comunicação às diferentes tarefas apresentadas. Com efeito, são encontradas no exame tarefas que o professor-candidato possivelmente executa em sala de aula, como é o caso da metalinguagem e da leitura de textos acadêmicos condizentes à área da Linguística Aplicada.

Adotamos a definição de tarefas elaborada por Nunan (1989), que aborda o termo a partir de uma visão interacionista. Sob esse ponto de vista, é aproximada à 
comunicação da sala de aula não só uma atividade interativa exterior ao contexto de ensino, como atividades realizadas no quotidiano, mas também atividades de percepção que englobem situações de uso da língua como instrumento de negociação e interpretação de significados.

O autor afirma existirem cinco componentes que formam uma tarefa, sendo eles: os objetivos que se pretende alcançar; o insumo que se oferece ao aluno como ponto de partida; as atividades que geram interação; o contexto dentro do qual as tarefas são realizadas; o papel do aluno e o papel do professor.

Para Nunan (1989), uma tarefa é "um trabalho de sala de aula que envolve os aprendizes em compreender, manipular, produzir ou interagir na língua-alvo enquanto sua atenção está focada no sentido e não na forma.” (p. 10)

Embora o autor defenda o uso de tarefas autênticas, que podem ser facilmente encontradas no cotidiano, fora da sala de aula, ele também acredita no valor de tarefas pedagógicas, que não são, necessariamente, repetidas no dia-a-dia, ou seja, não autênticas. O autor distingue essas tarefas autênticas e pedagógicas por meio da terminologia 'tarefas do mundo real' e tarefas pedagógicas. Considerando a definição de Nunan (1989), descrevemos as tarefas do teste de leitura e produção escrita do EPPLE.

A primeira tarefa do teste é baseada em um texto que aborda tópicos de Linguística Aplicada. Pede-se para que o candidato escreva um e-mail para um colega de trabalho discutindo o tópico apresentado no texto. Na segunda tarefa, o candidato deve responder a uma pergunta dissertativa, expressando também sua opinião sobre o tópico em discussão e, na terceira tarefa, deve escrever três perguntas referentes ao texto, como se elas fossem direcionadas aos seus alunos, com a intenção de avaliar as habilidades de leitura e escrita.

Na segunda parte do teste é apresentado um texto, supostamente escrito por um aluno, contendo vários erros gramaticais. São extraídas dez sentenças do texto e pede-se para que o candidato analise essas sentenças, aponte os possíveis erros e corrija-os caso eles realmente existam.

Na terceira e última parte do teste, são apresentados dois temas direcionados à área de ensino e aprendizagem de línguas, e pede-se ao candidato que escreva um texto dissertativo, discutindo e abordando o tema estabelecido. Vale ressaltar que todas as tarefas são apresentadas e devem ser realizadas em língua estrangeira, no caso do teste mencionado, a língua inglesa. 
Acreditamos que o formato do teste é bastante coerente ao objetivo que ele traz: o de não apenas avaliar proficiência, mas também o de utilizar tarefas voltadas para o contexto de ensino e aprendizagem de línguas, avaliando o desempenho pedagógico do professor.

No caso da primeira parte do instrumento, são abordados temas voltados à área de Linguística Aplicada, propondo uma discussão que abrange o universo do professor de línguas. Visto que o EPPLE é um exame para fins específicos, consideramos que suas tarefas apresentam uma abordagem específica, aquela que condiz ao meio de ensino e aprendizagem de línguas e ao fazer pedagógico do professor.

Como exemplo de tarefa para fins específicos, identificamos a de correção de erros, em que o professor identifica algum erro no texto escrito por um aluno e deve corrigi-lo. O candidato poderá ser avaliado nesse momento não apenas por identificar o erro, mas também por utilizar estratégias por meio das quais o erro é corrigido.

Considerando que a forma com que o professor aborda o tema identidade e diferença em sala de aula está diretamente ligada ao seu fazer pedagógico e ao ensino da língua estrangeira, utilizar uma tarefa no EPPLE que aborde essas questões e que leve o professor a refletir sobre elas. Seria bastante favorável àquilo que o teste busca avaliar, uma vez que identidade e diferença são aspectos primordiais para o ensino de línguas.

Ressaltamos ser necessário o despertar de consciência e de reflexão sobre o quanto importante para o ensino de língua estrangeira são a abordagem e o reconhecimento da identidade do outro, considerando os aspectos de pluralidade, diferença e diversidade que mencionamos no início dessa discussão.

Estudar uma língua estrangeira, discutindo e respeitando as diferentes identidades de forma a não segregá-las, mas, sim, vivê-las, é o caminho que nos leva a não só estudar uma língua estrangeira, mas a respeitar as diferentes identidades que existem nessa língua.

Sendo assim, concordamos com a visão não essencialista de identidade definida por Hall (1990) que foca não apenas as diferenças, mas também as características comuns e partilhadas tanto entre povos de etnias semelhantes como de etnias diferentes. Visão essa que vai ao encontro da necessidade de o professor de línguas abordar a cultura do outro por meio da diferença, que deve ser não apenas ser respeitada, mas também vivida/compartilhada. 
A partir do momento em que a identidade passa a ser vista por meio da diferença, passamos a compreender não só a identidade do outro, mas também a nossa própria identidade, que é também constituída por meio da diferença.

Concordamos com Levi (2007), quando ele afirma que, “A complexidade e a variação em nossa compreensão do conceito de cultura têm ecoado na série de abordagens, estratégias e técnicas que têm sido defendidas para o ensino de línguas e cultura”. (p. 104).

Embora o conceito de cultura seja complexo, é preciso priorizar sua dimensão e promover estratégias e técnicas efetivas para a sala de aula, com o intuito de despertar a consciência dos nossos aprendizes não apenas para a cultura do outro, mas para a própria cultura, levando-os a vivenciar a cultura do outro por meio da pluralidade e da vivência do que é diferente, uma vez que a nossa própria cultura é constituída por diversas identidades que se estabelecem por meio das diferenças. Dessa forma, não mais teremos culturas melhores ou piores, teremos culturas simplesmente diferentes.

\section{Considerações finais}

Em suma, ao considerarmos que um teste para professores de língua estrangeira tem como objetivo primordial ocasionar um efeito retroativo positivo, capaz de fazer com que seus candidatos busquem alcançar níveis de proficiência cada vez maiores, acreditamos que, ao inserirmos no teste de leitura e produção escrita do EPPLE uma tarefa que aborde o tema identidade e diferença, contribuiremos para que os professores pesquisem mais sobre o assunto, trabalhem mais tais questões com seus alunos, de forma a compreenderem melhor esses aspectos.

Sendo assim, baseados em uma tarefa a ser trabalhada em sala de aula apresentada por Levi (2007), propomos que seja inserida uma tarefa no teste de leitura e produção escrita do EPPLE que contemple o tema "identidade e diferença". A tarefa consistiria, por exemplo, na apresentação de dois e-mails escritos por alunos de língua inglesa de diferentes nacionalidades, abordando tópicos culturais. Ou seja, por meio do uso da língua inglesa, o aluno supostamente iria elaborar um e-mail discutindo um aspecto da sua própria cultura.

Baseado na leitura dos dois e-mails, o candidato deveria identificar quais características particulares de uma troca de e-mails podem levar à compreensão da cultura do outro, estabelecendo a identificação e compreensão da identidade do outro, pautadas nas diferenças. O objetivo da tarefa seria fazer com que o candidato escrevesse 
uma possível estratégia, que poderia ser utilizada em sala de aula, com o intuito de abordar a cultura dos dois estudantes, pautando-se na diferença e na pluralidade.

Acreditamos que, embora essa tarefa seja apenas uma amostra do que pode ser desenvolvido dentro da complexidade do tema "identidade e diferença", a tentativa de incorporá-la em um exame de proficiência destinado para professores de língua estrangeira contribuiria para que os candidatos ao exame buscassem refletir sobre o assunto, incorporando-o em sua pratica pedagógica.

Desse modo, considerando as reflexões de Silva (2011) que entende a linguagem, de forma mais geral, como um sistema se significação e como uma estrutura instável, afirmamos que a identidade e a diferença não podem ser compreendidas fora desses sistemas de significação nos quais adquirem sentido. O que objetivamos propor é que seria indispensável avaliar, por meio de uma tarefa que aborde o uso de língua estrangeira, questões de identidade e de diferença, que se constituem dentro desse sistema de significação, que é a linguagem.

Esperamos contribuir também para que todos os professores de língua estrangeira busquem refletir sobre o assunto de forma a incorporá-lo em sua prática pedagógica, considerando o mundo de pluralidade em que vivemos, onde não apenas o outro é diferente, visto que nossa própria identidade é pautada na diversidade, na pluralidade, na diferença.

Concluindo, acreditamos que o efeito retroativo do EPPLE poderá ser ainda mais positivo ao abordar aspectos de "identidade e diferença", visto que contribuirá para o aprimoramento da proficiência linguística, do fazer pedagógico e da reflexão sobre pluralidade, diversidade, diferença e identidade dos profissionais de língua estrangeira.

\section{REFERÊNCIAS}

CONSOLO, D. A.; TEIXEIRA DA SILVA, V. L. The TEPOLI test: Construct, updated tasks and new parameters to assess EFL teachers' oral proficiency. In: Anais do I Congresso Internacional da ABRAPUI. Belo Horizonte: UFMG, 2007. (CD-ROM).

HALL, S. Cultural identity and diaspora. In: RUTHER-FORD, J. (org.). Identity: community, culture, difference. Londres: Lawrence and Wishart, 1990.

NUNAN, D. Designing tasks for the communicative classroom. Cambridge: Cambridge University Press, 1989.

SCARAMUCCI, M. V. R. Proficiência em LE: considerações terminológicas e conceituais. Trabalhos de Linguística Aplicada 36, 2000, p. 11-22. 
SILVA, T. T. da. A produção social da identidade e da diferença. In: SILVA, T. T. da.; Hall, S.; WOODWARD, K. (Org.). Identidade e diferença: a perspectiva dos estudos culturais. Petrópolis - RJ: Vozes, 2011, p. 73 - 102.

WOODWARD, K. Identidade e diferença: uma introdução teórica e conceitual. In: SILVA, T. T. da.; Hall, S.; WOODWARD, K. (Org.). Identidade e diferença: a perspectiva dos estudos culturais. Petrópolis - RJ: Vozes, 2011, p. 73 - 102.

\section{Como referenciar este artigo}

ANCHIETA, Priscila Petian. Relações entre os conceitos "cultura, identidade e diferença" e o exame de proficiência EPPLE. Rev. EntreLínguas, Araraquara, v.3, n.1, p. 000-000, jan./jun. 2017. Disponível em: <https://doi.org/10.29051/rel.v3.n1.janjun.2017.9106>. E-ISSN: 2447-3529.

Submetido em: 28/11/2016

Aprovado em: 22/05/2017 